\title{
Clarification on the generation of absolute and potential vorticity in mesoscale convective vortices
}

\author{
R. J. Conzemius ${ }^{1,2}$ and M. T. Montgomery ${ }^{1,3}$ \\ ${ }^{1}$ Department of Atmospheric Science, Colorado State University, Fort Collins, Colorado, USA \\ ${ }^{2}$ WindLogics Inc., Grand Rapids, Minnesota, USA \\ ${ }^{3}$ Naval Postgraduate School, Monterey, California, USA \\ Received: 18 February 2009 - Published in Atmos. Chem. Phys. Discuss.: 23 March 2009 \\ Revised: 16 September 2009 - Accepted: 17 September 2009 - Published: 12 October 2009
}

\begin{abstract}
In this paper, we clarify several outstanding issues concerning the predominant mechanism of vorticity generation in mesoscale convective vortices (MCVs) in weak to modest baroclinic environments with nonzero Coriolis parameter. We examine also the corresponding diabatic heating profiles of the convective and stratiform components of the MCS and their effects on the concentration and dilution of PV substance.
\end{abstract}

\section{Introduction}

In their pioneering paper outlining a theory for the maintenance of long-lived mesoscale convective systems (MCS)s, Raymond and Jiang (1990) stated that "the convective and anvil regions have the same qualitative effect on potential vorticity distributions, i.e. potential vorticity in the lower half of the troposphere is increased, whereas potential vorticity near the tropopause is decreased". With regard to the absolute vorticity generation in mesoscale convective vortices (MCVs), it is commonly thought that, because the vortex resides in an area of stratiform precipitation, the stratiform processes must be largely responsible for generating its absolute vorticity (Hertenstein and Schubert, 1991; Johnson and Bartels, 1992; Fritsch et al., 1994; Davis and Trier, 2002).

"This large derivative of the heating, coupled with the longer influence time associated with the width of the stratiform region, allows the potential vorticity signature of the

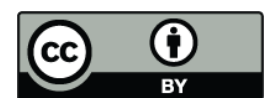

Correspondence to: R. J. Conzemius (robert.conzemius@att.net) stratiform region to dominate over the signature of the convective line" (Hertenstein and Schubert, 1991).

"Intensification of the MCV began overnight when a lower-tropospheric mesoscale vortex formed on the northern end of the north-south-oriented convective line. Intensification of the midtropospheric vortex followed, occurring in response to the development of a stratiform precipitation region" (Davis and Trier, 2002).

A number of these studies have indeed provided partial answers to the questions regarding the generation mechanism of absolute vorticity in the MCV. However, as Raymond and Jiang stated, "further work is needed in determining the contribution from each effect in a variety of mesoscale systems." The purpose of this paper is to take a more detailed look at the vorticity generation mechanisms, both in terms of PV and absolute vorticity. In particular, we will take a look at the relative contributions to the PV concentration from the convective and stratiform regions of two simulated MCSs.

In terms of the absolute vorticity budget, the primary intensification mechanism is by means of the thermally direct circulation driven by diabatic heating within the convective system and the associated flux convergence of planetary and relative vorticity. When referring more directly to potential vorticity $(\mathrm{PV})$, the intensification can be explained (at least partially, when viewed in geometric coordinates, using the material form of the PV equation) in terms of the vertical gradient of diabatic heating within the convective system. In either case, diabatic heating is required, whether it be by driving the thermally direct circulation or producing PV by vertical gradients.

The aim of this paper is to resolve a number of ambiguous conclusions regarding the sources of PV in MCVs. In their satellite-based study of MCVs, Bartels and Maddox (1991)

Published by Copernicus Publications on behalf of the European Geosciences Union. 
concluded that "the rapid mesovortex generation observed can be explained by the stretching term of the vorticity equation." This conclusion was limited to those MCVs for which a visually recognizable signature existed in satellite imagerypreferentially in weak shear. Seven years later, Weisman and Davis (1998) argued that "for systems that develop in an environment with weak-to-moderate shear, a line-end vortex pair is generated primarily via the tilting of horizontal vorticity generated within the system-scale cold pool" and that "convergence at midlevels enhances Coriolis rotation over the longer term, leading to the preferred development of a cyclonic vortex". These conclusions certainly apply for the storms in those simulations. However, the conclusions may not apply to all storms as they form in a wide variety of background conditions. For example, storms form in environments with vastly different thermodynamic and kinematic profiles, and they also evolve in response to atmospheric motions over a broad range of scales. It can be thought that the complex array of possible influencing factors in storm development would broaden the spectrum of development characteristics for MCVs beyond the traditional "tilting then stretching" argument.

This wider range of possibilities applies both to the budgets of absolute vertical vorticity and to the respective roles of the convective and stratiform precipitation regions of MCSs. Regarding the former, for example, some studies have concluded that tilting of vorticity is the primary contributor to the formation of MCVs (Skamarock et al., 1994; Weisman and Davis, 1998), but others have found vortex tube stretching to be the primary vorticity generation mechanism at mid-levels (Brandes, 1990; Bartels and Maddox, 1991; Johnson and Bartels, 1992; Davis and Trier, 2002), and still others have suggested that both mechanisms may be important at different stages of the MCV life cycle (Cram et al., 2002) or at different scales of atmospheric motion (Knievel and Johnson, 2003). Although these studies may appear to be in conflict with each other, they have been carried out in different conditions using different analysis techniques and different model domain sizes and resolutions, so the evolution need not be the same in all cases. Kirk (2007) made a substantial effort to resolve the apparent confusion among all these studies by using a phase plot method to track the evolution of vertical vorticity budgets in different MCVs. To add to the collection of cases that have been observed and simulated, and with the intent of providing some clarification of the MCV formation process as it may occur in moist baroclinic, mid-latitude environments, we perform here detailed analyses of the circulation budget of two simulated MCVs over their lifetime, from genesis to maturity. Additionally, our idealized simulations will start with a basic state in thermal wind balance and include the effects of larger scale baroclinic environments, something which was not included in the Davis and Weisman (1994), Skamarock et al. (1994), Weisman and Davis (1998), or Cram et al. (2002) simulations.
As a part of this goal, we will provide a qualitative overview of the PV development process and the aggregation process of $\mathrm{PV}$ elements in order to make a cursory investigation into the role of $\mathrm{PV}$ concentration on the storm scale (small, intense, convective updrafts) as well as on the MCV scale. Such a look is intended to qualitatively illustrate the processes behind the more quantitative budgets that appear earlier in the paper, highlighting the role of vorticity on smaller scales, which is primarily generated by tilting mechanisms, on the development of vorticity at the larger, MCV scale as well as the flux convergence of planetary vorticity and relative vorticity on the system-wide scale. Given the linear hodographs employed in all basic states used to date in idealized studies of MCV dynamics, one would expect tilting to generate positive and negative relative vorticity centers in nearly equal amounts (e.g., Rotunno, 1981). When tilting occurs in an environment with horizontal vorticity, due to a convective updraft, centers of positive and negative vorticity are produced. When vertical gradients of diabatic heating act upon these vorticity centers, they produce a concentration of PV substance (e.g. Tory et al., 2007) in mid-levels and a dilution of PV substance above, resulting in an approximate vertical stacking of positive and negative PV anomalies, respectively. The evolution of these positive and negative PV centers can be monitored in numerical simulations to discover their relative contributions to the MCV PV.

The magnitude of convective versus stratiform processes contributing to the PV concentration in MCVs is less well understood. Many studies attribute the PV concentration to diabatic heating gradients that are present in the stratiform precipitation region of an MCS (Hertenstein and Schubert, 1991; Johnson and Bartels, 1992; Fritsch et al., 1994; Davis and Trier, 2002). This traditional view is supported by the fact that MCVs are consistently observed to develop in the stratiform portion of MCSs. However, the diabatic heating profiles presented by Houze $(1997,2004)$ imply that the PV concentration may come from either convective or stratiform processes. A look at the conceptual model of the MCS, such as presented in Fig. 14 of Houze (2004), with air ascending in convective elements at the leading edge of the MCS then moving rearward to the stratiform precipitation region, reveals that the PV concentration in stratiform and convective areas of the MCS are part of the same process and that perhaps the PV concentration really need not be separated into stratiform and convective components. Nevertheless, MCSs do have varying amounts of convective and stratiform precipitation within them, and the purpose of our analysis is to provide evidence that convective processes may be a more substantial contributor to PV concentration than has been traditionally thought.

Deep convection often occupies a relatively small area (by percentage) of an MCS, but the diabatic heating rates and vertical mass flux per unit area are at least an order of magnitude greater, locally, in convective than in stratiform 
Table 1. Selected parameters for simulations.

\begin{tabular}{|c|c|c|c|c|}
\hline & & & Control & CAPE \\
\hline \multirow[t]{5}{*}{ Basic State } & $\mathrm{T}_{\text {cent }}(\mathrm{K})$ & & 293 & 303 \\
\hline & Shear ${ }_{\text {cent }}\left(\mathrm{m} \mathrm{s}^{-1} \mathrm{~km}^{-1}\right)$ & & 1.5 & 1.5 \\
\hline & Width $_{B Z}$ (degrees) & & 60 & 60 \\
\hline & $\mathrm{CAPE}_{\text {cent }}\left(\mathrm{J} \mathrm{kg}^{-1}\right)$ & & 0 & 2247 \\
\hline & $\mathrm{CAPE}_{\max }\left(\mathrm{J} \mathrm{kg}^{-1}\right)$ & & 0 & 4200 \\
\hline \multirow[t]{6}{*}{ Inner domains } & Start time (hours) & & 120 & 120 \\
\hline & Move frequency (hours) & D3 & - & 72 \\
\hline & & D4 & 24 & 8 \\
\hline & Grid dimensions & $\mathrm{D} 2$ & $94 \times 76$ & $154 \times 94$ \\
\hline & & D3 & $229 \times 172$ & $289 \times 199$ \\
\hline & & D4 & $271 \times 211$ & $289 \times 253$ \\
\hline Initial vortex radial profile & & & Guassian & Isolated \\
\hline
\end{tabular}

precipitation. Additionally, the early stages of the life cycle of many MCSs tend to be dominated by convection, leading to the distinct likelihood that, although MCVs are rarely (if ever) observed in the convective region of an MCS, convection can be the primary contributor to the development of PV anomalies that are eventually revealed in the stratiform precipitation area. Again, since most of the mass flux is processed through both the leading convective region and the stratiform precipitation, it can be argued that both may be similar contributors. The present study will clarify this issue by performing a detailed analysis of the diabatic heating profiles. These diabatic heating profiles should reveal the quantity of mass processed through the convective system since diabatic heating rate is proportional to the mass flux.

\section{Simulations}

The simulations were part of an investigation into the mechanisms for the development of MCVs in weak but non-trivial baroclinic environments by Conzemius et al. (2007) and are further described in that study. The goal of the original study was to conduct idealized simulations capable of coarsely resolving deep convective processes in an environment with relatively weak shear, whereby the dynamics of MCV maintenance and growth could be examined, but still encompassing an area large enough that permits larger scale baroclinic development as part of the MCV lifecycle.

For this study, we conducted two simulations, the primary difference between which was the value of ambient CAPE. The first simulation was designated the control simulation and started with a basic state thermodynamic profile that was neutral to the moist ascent of boundary layer air parcels. By definition, this neutral atmosphere had no CAPE, but CAPE developed during the simulation, reaching maximum values of around $700 \mathrm{~J} \mathrm{~kg}^{-1}$. The second simulation had $2000 \mathrm{~J} \mathrm{~kg}^{-1}$ CAPE in the center of the baroclinic zone and was simply designated as the CAPE simulation.
We performed both simulations using the MM5 model (Grell et al., 1994), which is a limited area, non-hydrostatic, sigma coordinate model. We performed the simulations on an $11000 \mathrm{~km}$ by $5000 \mathrm{~km}$ domain using an outer, coarseresolution grid with a $90 \mathrm{~km}$ grid interval and three nested inner grids whose intervals were 30,10 , and $3.3 \mathrm{~km}$. The initial state consisted of an Eady-type baroclinic background, in which all variables are a function of latitude and pressure only. The initial state differs from the Eady basic state, however, in that a stratosphere was included, and the shear was half the value of the Eady basic state. The lateral boundary conditions were prescribed to be the initial, basic state conditions, the upper boundary condition was a gravity wave radiation condition, and the bottom boundary conditions were no slip but free of sensible or latent heat fluxes. Additionally, there was no infrared or shortwave radiation. Without heat fluxes or radiation, there was no diurnal cycle in the experiments. Further details and reasoning behind these experiments are provided in Conzemius et al. (2007). The basic parameters of the simulations are listed in Table 1.

In order to initiate an MCS, we inserted a warm core, low-level vortex, with zero PV perturbation in its interior (to avoid preconditioning the simulation with interior PV) into the background state. The vortex had a radial profile of potential temperature perturbation with maximum magnitude of $3.0 \mathrm{~K}$ at the surface and decayed with height. The radial profile was in the shape of a Gaussian function for the control simulation and in the shape of the second derivative of the Gaussian profile (i.e. "isolated") for the CAPE simulation. As it turns out, the primary effect of the initial vortex in the simulations, as discussed in Conzemius et al. (2007), is to excite a spectrum of moist baroclinic waves. The MCS develops just to the east of the surface low that is associated with the most rapidly amplifying baroclinic wave. The isolated profile was chosen for the CAPE simulation in an attempt to minimize the impact of the moist baroclinic instability. However, since the MCS development inevitably 
was tied to the baroclinic processes, this change served only to slow the large scale moist baroclinic growth and thereby delay the MCS development.

Conzemius et al. (2007) describe the evolution of these simulations in greater detail, so we will only summarize here. In the control simulation, an MCS developed approximately $126 \mathrm{~h}$ into the simulation, within an intensifying, moist baroclinic cyclone. An MCV developed within the MCS, and the MCS precipitation gradually became more stratiform in nature as the simulation proceeded. In the CAPE simulation, the MCS developed approximately $150 \mathrm{~h}$ into the simulation (this was delayed due to the choice of an isolated initial vortex temperature radial profile), but when the growth occurred, it was much more rapid and extensive. The MCV that developed was much stronger as well, with the precipitation shield remaining convective much longer into the simulation.

\section{Diagnostic calculations}

\subsection{Vorticity budget calculations}

Similar to the procedure expounded in Haynes and McIntyre (1987) and the methodology outlined in Weisman and Davis (1998), Cram et al. (2002) and Davis and Trier (2002), we use the flux form of the vorticity equation and calculate areaand temporal-averages of the circulation tendency:

$$
\frac{\partial \zeta_{a}}{\partial t}=-\frac{\partial}{\partial x}\left(u \zeta_{a}+\omega \frac{\partial v}{\partial p}-G\right)-\frac{\partial}{\partial y}\left(v \zeta_{a}-\omega \frac{\partial u}{\partial p}+F\right)
$$

where $\zeta_{a}$ is the absolute vorticity defined as $\zeta_{a}=f+\partial v / \partial x-\partial u / \partial y$, and $F$ and $G$ represent the horizontal components of the effects of additional forces, such as friction, which are neglected in this analysis for simplicity. The neglect of frictional forces can be justified by the fact that the initial near-surface winds are very weak and is further supported by the comparisons between vorticity budget profiles and actual vorticity change presented in Sect. 4.1.

In addition to avoiding the inherent problem of measuring the small difference between large terms that can occur when using the material form of the vorticity equation (Haynes and McIntyre 1987), the strength of this method lies in the fact that, through Gauss's theorem, the tendency of the circulation for any enclosed area fixed in space can be written in terms of the line integral of the flux component normal to the boundary of the area (Davis and Trier, 2002):

$$
\frac{\partial C}{\partial t}=\iint_{A} \frac{\partial \zeta_{a}}{\partial t}=-\oint\left(\boldsymbol{u} \zeta_{a}-\omega \hat{\boldsymbol{k}} \times \frac{\partial \boldsymbol{u}}{\partial p}\right) \cdot \hat{\boldsymbol{n}} d l
$$

where $C$ is the absolute circulation, $A$ represents the area encompassed by the closed loop, and $\boldsymbol{u}$ the horizontal velocity. We will refer to the first term under the integral as the horizontal convergence of absolute vorticity (horizontal flux term). A comparison between the flux and the material forms of the vorticity equation reveals that the horizontal flux term in Eq. (2) is equivalent to the horizontal advection term plus the stretching term in the material form of the vorticity equation. The second term is an amalgamation of the vertical advection of vertical vorticity and the tilting of horizontal vorticity into vertical vorticity and will be hereafter denoted simply as the tilting-like term (see Tory et al. (2007) for clarification of this terminology). For comparison, Davis and Trier (2002) refer to these terms as stretching and tilting, respectively.

The corresponding form of the PV equation is most conveniently written in theta (potential temperature) coordinates:

$$
\iint_{A} \frac{\partial(\sigma Q)}{\partial t}=-\oint\left(\boldsymbol{u} \sigma Q-\dot{\theta} \hat{\boldsymbol{k}} \times \frac{\partial \boldsymbol{u}}{\partial \theta}\right) \cdot \hat{\boldsymbol{n}} d l,
$$

where $Q$ is $\mathrm{PV}\left(\mathrm{m}^{2} \mathrm{~s}^{-1} \mathrm{~K} \mathrm{~kg}^{-1}\right), \sigma$ the isentropic density $\left(\mathrm{m}^{-2} \mathrm{~K}^{-1} \mathrm{~kg}\right)$, and $\dot{\theta}$ the material rate of change of potential temperature. In theta coordinates, the $\mathrm{PV}$ substance $\sigma \mathrm{Q}$ is simply $\partial v / \partial x-\partial u / \partial y+f$, so one can see a striking similarity between Eqs. (2) and (3) (the zonal and meridional partial derivatives of course being taken on surfaces of constant pressure and potential temperature, respectively). Tory et al. (2007) and Haynes and McIntyre (1987) discuss at length the corresponding PV equation in differential form. The divergence of the second (non-advective) flux term can be split into two terms, one of which describes tilting-like effects and the other describing vertical advection-like effects. The tilting-like and vertical advection-like terms tend to largely balance each other, typically leaving the first term in the line integral as the dominant term on the right hand side of Eq. (3). Thus, we would expect an increase in PV substance over any area to be mostly due to flux convergence (i.e. a concentration) of PV. This concentration of PV substance occurs mostly at low levels, driven by diabatic heating within the MCS. At upper levels, we would expect a negative PV anomaly to form as flux divergence dilutes the PV substance.

\subsection{Areas and times of analysis on innermost grid}

The rectangle forming the contour of integration was chosen to be as close to the vortex center while encompassing all significant cyclonic relative vorticity. In practice, the southern line segment was placed along the maximum $850-700 \mathrm{hPa}$ rear inflow into the convective system, while the western and northern line segments were placed as close as possible to the cyclonic vorticity maximum but in regions encountering little or no convection. To allow for the movement of the convective system within the averaging time interval, we placed the eastern line segment far enough ahead of the convective line that the precipitation did not advance beyond the eastern line until after the end of the averaging time interval.

Four time periods, each of 288 min duration, were chosen for the vorticity budget analysis of the control simulation. 
Table 2. Analysis box characteristics.

\begin{tabular}{lcccr}
\hline Simulation & & Time interval (h) & Dimensions (grid cells) & Area $\left(\mathrm{km}^{2}\right)$ \\
\hline Control & A & $124.8-129.6$ & $65 \times 45$ & 32500 \\
& B & $129.6-134.4$ & $110 \times 60$ & 73300 \\
& C & $134.4-139.2$ & $140 \times 70$ & 109000 \\
& D & $170.4-175.2$ & $175 \times 110$ & 214000 \\
CAPE & A & $152-156$ & $100 \times 56$ & 62200 \\
& B & $156-160$ & $140 \times 110$ & 171000 \\
& C & $160-164$ & $145 \times 130$ & 209000 \\
& D & $168-172$ & $230 \times 160$ & 409000 \\
\hline
\end{tabular}

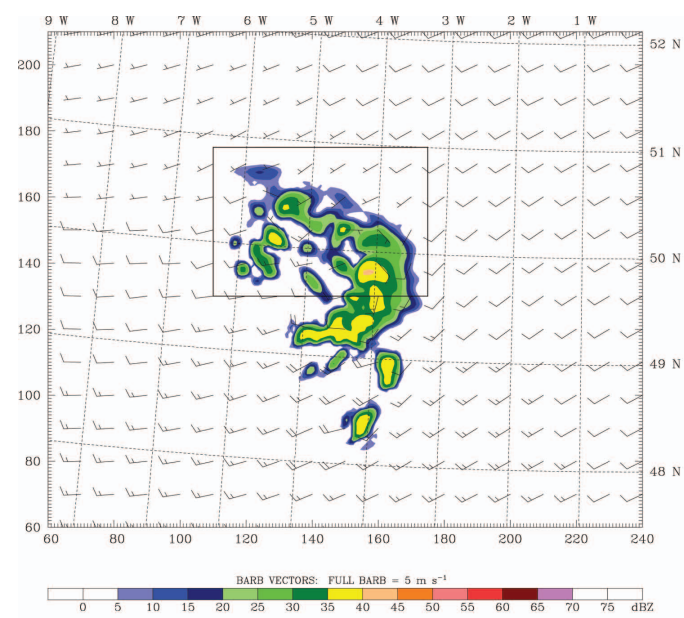

(a)

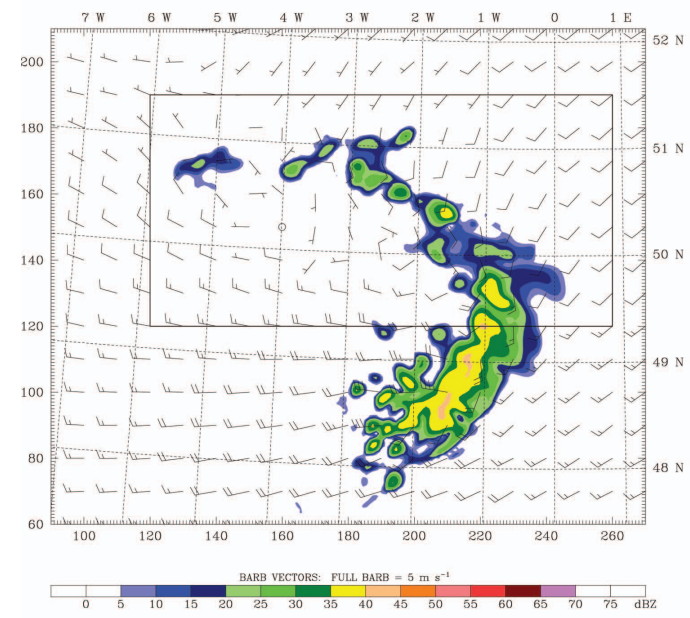

(c)

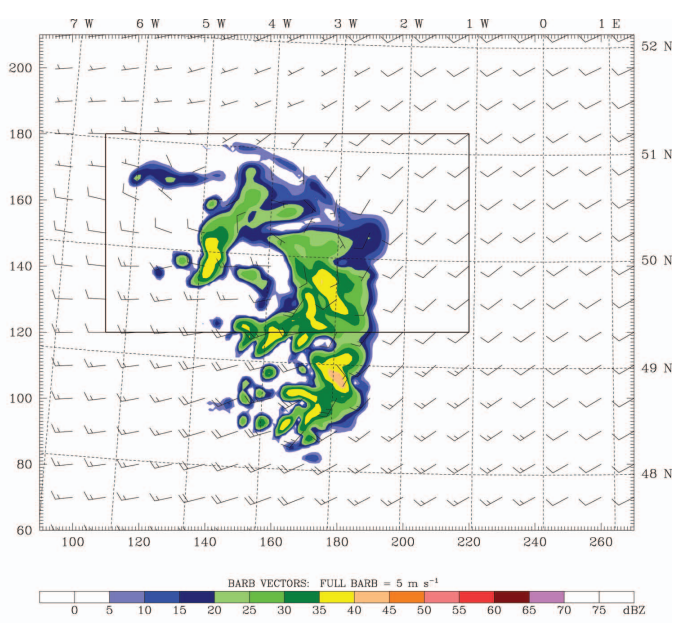

(b)

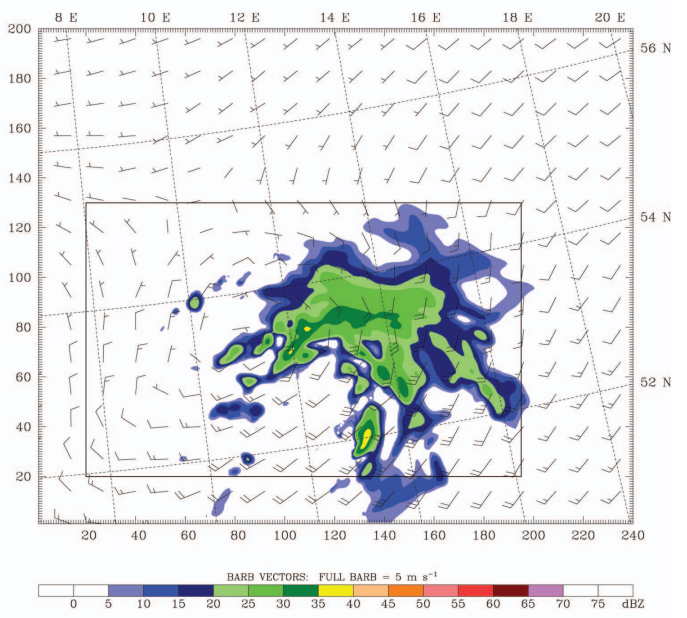

(d)

Fig. 1. Model-derived reflectivity (dBz; shading), horizontal flow (barbs; knots) at $700 \mathrm{hPa}$, and analysis boxes at the following times in the control simulation: (a) $129.6 \mathrm{~h}$; (b) $132.0 \mathrm{~h}$; (c) $136.8 \mathrm{~h}$; and (d) $172.8 \mathrm{~h}$. The equivalent radar reflectivity factor for rain, $Z_{e}\left(\mathrm{~mm}^{6} \mathrm{~m}^{-3}\right.$ ), is computed following Fovell and Ogura (1988) as $Z_{e}=720 \alpha k N_{o} \lambda^{-7}\left(\rho / \rho_{w}\right)^{2}$, where $\alpha$ is the nondimensional ratio of backscattering for the reflecting substance ( 1 for water, 0.213 for ice), $k=10^{18}$ is the conversion factor from $\mathrm{m}^{6}$ to the units of $Z_{e}, N_{o}$ is the intercept parameter of the raindrop size distribution, $\rho$ the density of the substance of interest (water or ice), and $\rho_{w}$ the density of water. $\lambda$ is the slope of the raindrop size distribution: $\lambda=\left(\pi \rho_{w} N_{o} / \rho_{a} q_{r a}\right)^{1 / 4}$, where $\rho_{a}$ is the density of dry air, and $q_{r a}$ the rainwater mixing ratio. The reflectivity is expressed in decibels, or $\mathrm{dB} Z$, where $\mathrm{dB} Z=10 \log _{10}(Z e)$. 


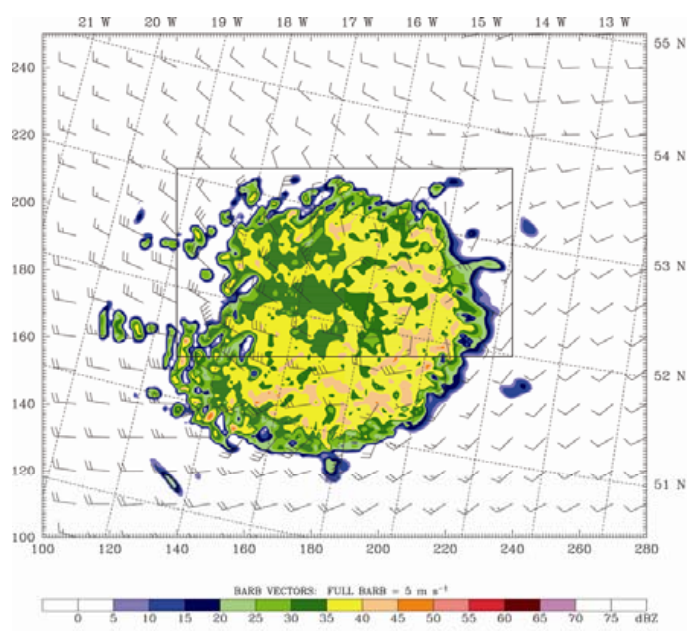

(a)

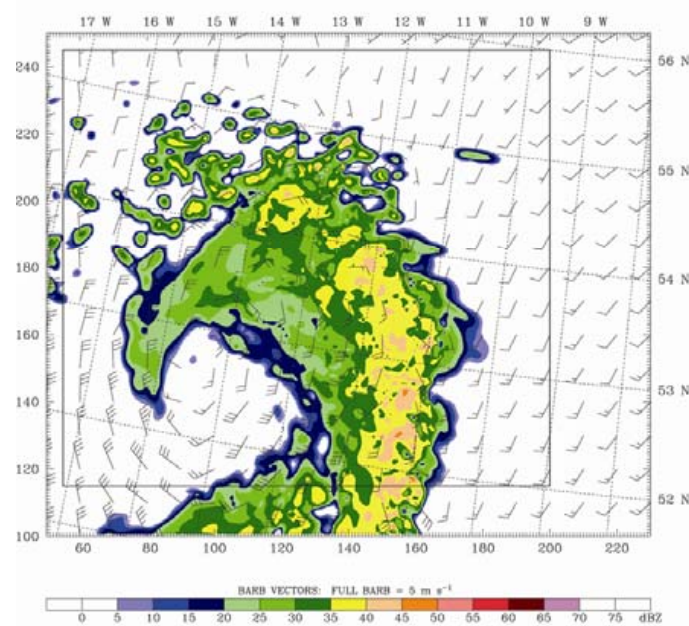

(c)

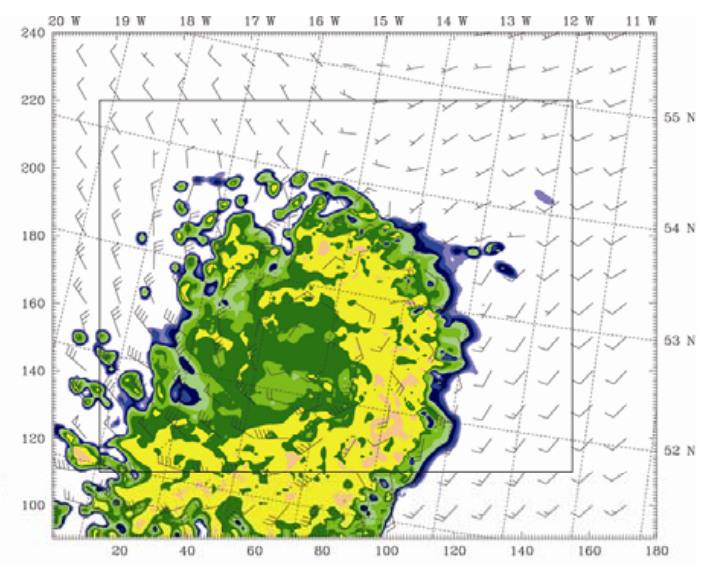

(b)

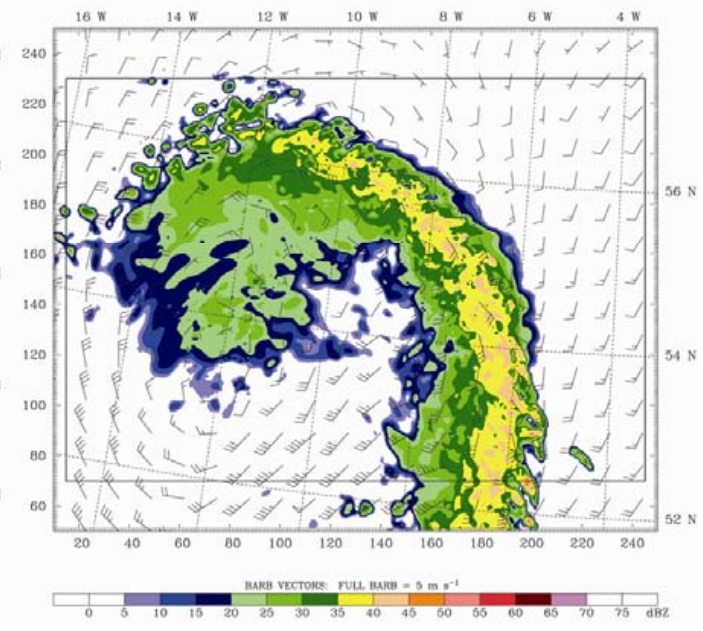

(d)

Fig. 2. Model-derived reflectivity (dBz; shading), horizontal flow (barbs; knots) at $700 \mathrm{hPa}$, and analysis boxes at the following times in the CAPE simulation: (a) $156 \mathrm{~h}$; (b) $158 \mathrm{~h}$; (c) $162 \mathrm{~h}$; and (d) $170 \mathrm{~h}$.

The chosen time periods encompass 1 . initiation and organization of deep convection accompanied by intensification of the baroclinic cyclone, 2. expansion of the stratiform precipitation region and slight weakening of convection (very little deepening occurs during this phase), 3. a period of essentially no deepening of the baroclinic cyclone, and 4. a later period, characterized by a resumption of deepening. The line integral was calculated at each model level on the innermost $3.3-\mathrm{km}$ domain at $8 \mathrm{~min}$ intervals, and time averaging was performed over the 288-min analysis period.

For the CAPE simulation, the time averaging periods were $240 \mathrm{~min}$ each. The first period corresponds to the development and organization of the MCS and intensification of the baroclinic cyclone. The next two periods correspond to the continued expansion of the stratiform precipitation area, during which time the baroclinic cyclone did not deepen (at least in terms of surface pressure) significantly. The final period saw a resumption of rapid deepening of the baroclinic cyclone.

The placement of the analysis boxes is shown for the control simulation in Fig. 1 and for the CAPE simulation in Fig. 2. In Table 2, we provide information about the characteristics of the analysis boxes. Because of the increasing size of the circulating flow in the simulations, we increased the size of the analysis box between successive analysis intervals in order to encompass the cyclonic relative vorticity.

\subsection{Convective versus stratiform partitioning method}

By partitioning the updraft mass flux into a convective and a stratiform portion, one can assess the contributions of convective and stratiform areas to the concentration of absolute vorticity or PV within the system. The convective-stratiform partitioning method we used is similar to that used in Tao 
et al. (1993) and Braun (2009) except that the thresholds for defining convective areas are somewhat higher. The partitioning was done on a two-dimensional, grid column basis. Grid columns were designated convective if the surface precipitation rate was twice the average precipitation rate of the immediate, surrounding four cells. In such cases, the surrounding four cells were also designated as convective. Secondly, all grid cells with a rainfall rate exceeding $25 \mathrm{~mm} \mathrm{~h}^{-1}$ were assigned to the convective bin. In some situations, strong convective updrafts may not have rain reaching the surface, so if vertical velocity at any grid point in the column exceeded $5 \mathrm{~m} \mathrm{~s}^{-1}$, or if the cloud liquid water content exceeded $0.5 \mathrm{~g} \mathrm{~kg}^{-1}$ below the freezing level, then the column was designated as convective. For the remaining (nonconvective) grid columns, stratiform areas were defined as those with a precipitation rate of at least $0.1 \mathrm{~mm} \mathrm{~h}^{-1}$. Grid cells within the analysis box meeting none of these criteria were assigned to the non-precipitating category. The areas and time periods of analysis were the same as for the vorticity budget analysis.

Since the tendency of area average potential vorticity substance is driven by the horizontal flux convergence of PV substance (Eq. 3), and because the absolute vorticity tendency is also affected by horizontal flux convergence (Eq. 2), one can invoke mass conservation to relate the flux convergence of either quantity to the updraft mass flux within the MCS. Since the vertical mass flux is directly related to the diabatic heating rate in the system (we assume that the diabatic heating rate is essentially balanced by the upward transport of smaller potential temperature), it is equally useful to use diabatic heating rate as a proxy for mass flux. Our initial calculations demonstrated that the mass flux profiles (not shown) were qualitatively very similar to the profiles of diabatic heating rate. Similar to Kirk (2007), the diabatic heating rate was calculated by taking the residual of the thermodynamic equation (time tendency minus advection) and averaging over horizontal planes within the analysis area. We assumed diffusion effects on the thermodynamic equation to be negligible.

\section{Sources of absolute vorticity and PV}

\subsection{Vorticity budget profiles}

During most of the time periods of our analysis, the tiltinglike term is a contributor to the creation of positive vertical vorticity in the lower troposphere up to about $700 \mathrm{hPa}$, but its calculated contribution is sensitive to the placement of the rectangle used to calculate the loop integrals. The tilting-like term generally becomes large during brief intervals when the southern line segment intersects individual convective cells, which make strong contributions to the vorticity budget on scales smaller than the size of the MCS itself (Knievel and Johnson 2003). The four hour integration time smoothes out these contributions, however. Analyses on the coarser grids (not shown), which do not resolve individual convective cells, reveal similar-looking profiles of vorticity budget terms, suggesting some MCS scale tilting as well. Nevertheless, the circulation budget during the earliest analysis period (Fig. 3a) is dominated by the horizontal flux convergence of absolute vorticity. This finding refutes the "tilting then stretching" argument proposed by Weisman and Davis (1998) for this particular case. As discussed in the introduction, the initial state differs from the Weisman and Davis (1998) simulations due to its zero initial CAPE and its inclusion of baroclinic processes.

This finding is also somewhat different from that of Kirk (2007), where the tilting-like term (vertical momentum flux curl) contributed most strongly in the mid-troposphere. The analysis technique we employed here, however, is different from that used in Kirk (2007), and consequently, it is rather difficult to attribute the same meaning to the results. In particular, our analysis box was fixed in time, whereas in Kirk (2007), the box moves with the MCV. Additionally, the Kirk analysis box is $72 \mathrm{~km} \times 72 \mathrm{~km}$ (an area of $5184 \mathrm{~km}^{2}$ ), whereas our analysis boxes ranged in size from $32500 \mathrm{~km}^{2}$ to $409000 \mathrm{~km}^{2}$.

On the scale of the MCS, the horizontal flux convergence is, by far, the dominant mechanism responsible for the spin up of mid-level cyclonic absolute vorticity. The horizontal flux convergence in Fig. 3a and b is strongest at low levels, consistent with a divergence profile associated with deep convection (Houze 1997) and also consistent with the findings of Kirk (2007).

By $t=129.6 \mathrm{~h}$ (Fig. 3b), a closed circulation is evident at $700 \mathrm{hPa}$ (not shown). Between $t=124.8$ and $t=134.4 \mathrm{~h}$ into the simulation, the mid-level circulation strengthens substantially. This pattern is consistent with the re-intensification of the 27-28 May 1998 MCV investigated by Davis and Trier (2002). The stratiform precipitation is also expanding rapidly, and during this time, the horizontal flux convergence of absolute vertical vorticity is reflective of the stratiform divergence profile suggested by Houze (1997), with its flux convergence maximum at higher altitude than in Fig. 3a. In the ensuing time period (Fig. 3c), the circulation tendency is strongly positive in the upper troposphere, due to the horizontal flux divergence of negative relative vorticity.

At a much later time (Fig. 3d), when the surface low undergoes a steadier strengthening, the circulation budget is almost entirely dominated by the horizontal flux term. The dominance of this term shows that the mean secondary circulation associated with the vortex (convergence at low levels, rising in the vicinity of the vortex, and divergence at upper levels) drives the strengthening of the circulation, but this result might also be an artifact of the analysis area. Due to the size and orientation of the MCS, it was difficult to place the southern portion of the line integral along the maximum rear inflow into the system. The system is also somewhat less 
(a) $t=124.8 \mathrm{~h}$ to $t=129.6 \mathrm{~h}$

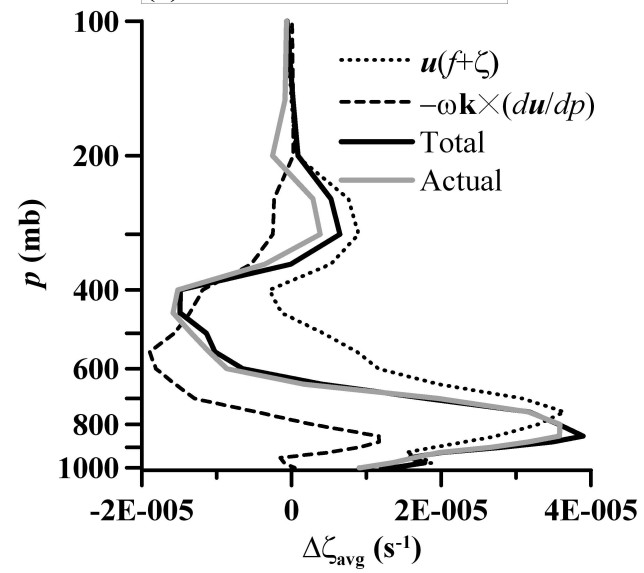

(c) $t=134.4 \mathrm{~h}$ to $t=139.2 \mathrm{~h}$

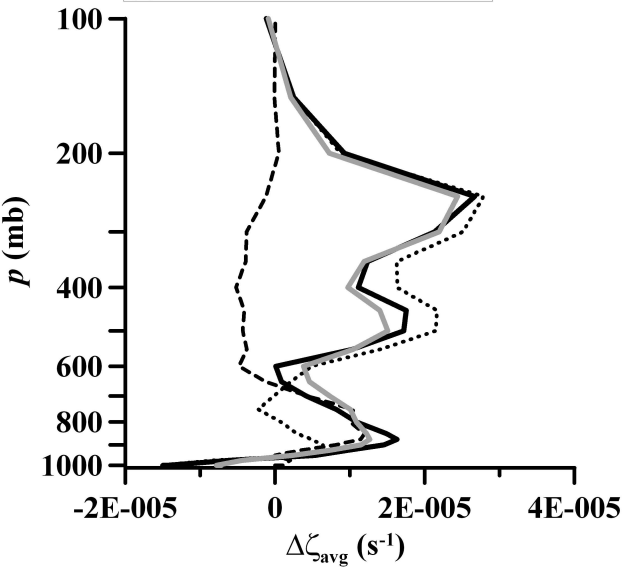

(b) $t=129.6 \mathrm{~h}$ to $t=134.4 \mathrm{~h}$

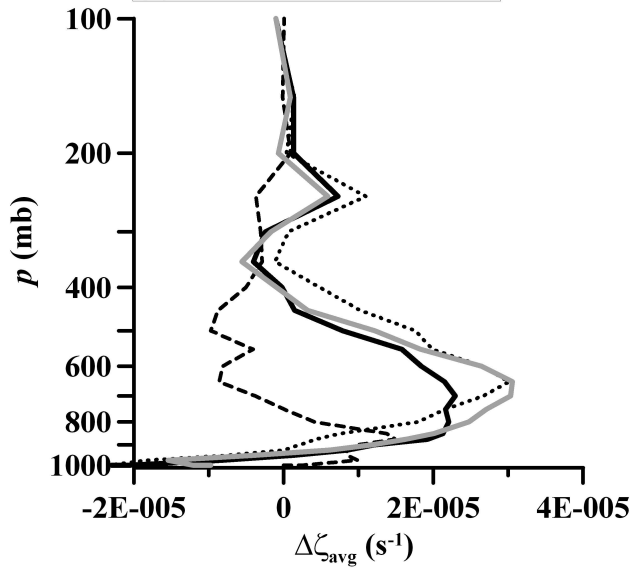

(d) $t=170.4 \mathrm{~h}$ to $t=175.2 \mathrm{~h}$

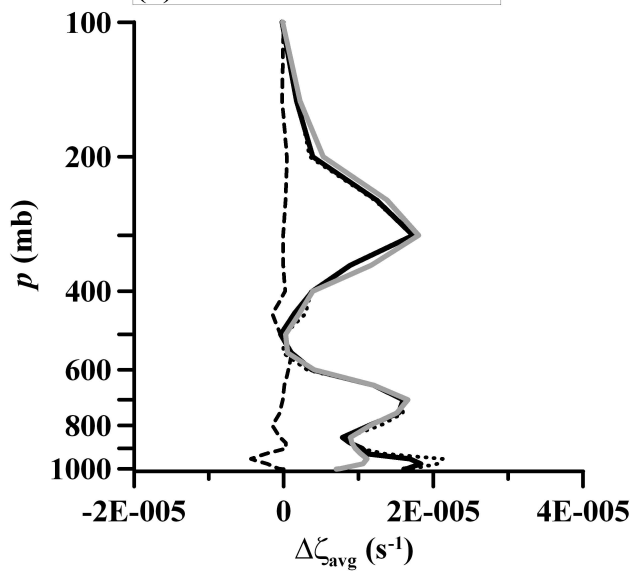

Fig. 3. Circulation budget analysis expressed as area average vorticity change for a rectangle surrounding the vortex in the control simulation on the $3.3-\mathrm{km}$ grid for the time periods: (a) $124.8 \mathrm{~h}$ to $129.6 \mathrm{~h}$; (b) $129.6 \mathrm{~h}$ to $134.4 \mathrm{~h}$; (c) $134.4 \mathrm{~h}$ to $139.2 \mathrm{~h}$; and (d) $170.4 \mathrm{~h}$ to $175.2 \mathrm{~h}$, respectively. The dotted line indicates the line integral of horizontal flux convergence of absolute vorticity; the dashed line is the corresponding, tilting-like term; the solid black line is the total of the horizontal and tilting-like terms; and the solid gray line is the actual change over the period of integration.

convective at this time. Section 4.2 will explore the convective and stratiform partitioning in greater detail.

The profiles of the vorticity budget terms at early times (Fig. 3a, b) resemble those in Davis and Galarneau (2009), particularly with regard to the peak of flux convergence in the middle troposphere and the dominance of flux convergence in general (N.b. our flux convergence term is the sum of the eddy flux and stretching terms defined in Davis and Galarneau). In both analyses, the budget terms were calculated using rectangles of similar size, the MCVs occurred within similar values of deep layer shear, and baroclinic effects were included in the simulations. One particular difference in the vorticity budget terms, however, lies in the tiltinglike term in the lower troposphere, which is negative in the Davis and Galarneau analyses but positive in ours. This difference may be due to the eastern and northern portions of the box being located in precipitation areas in the Davis and
Galarneau cases. Further analysis is required to uncover the root of these differences, but the overall result is the same. That is, tilting is secondary to stretching in the production of absolute vorticity.

In the CAPE simulation (Fig. 4), the evolution of the vorticity budget is somewhat different. In the initial time period (Fig. 4a), the horizontal flux convergence term is responsible for all the spin-up of absolute vorticity below the $500 \mathrm{hPa}$ level, except for the lowest $100 \mathrm{hPa}$ (where cold pool effects occur - see below). Once again, the overall profile and dominance of the flux convergence term is similar to the results of Davis and Galarneau (2009) during this initial analysis period. Thereafter (Fig. 4b and c), the flux convergence term actually becomes a negative contributor to the absolute vorticity below about $600 \mathrm{hPa}$, and the spin up of absolute vorticity is provided solely by the tilting-like term. It appears that the cold pool generation is influencing the flux convergence 
(a) $t=152 \mathrm{~h}$ to $t=156 \mathrm{~h}$

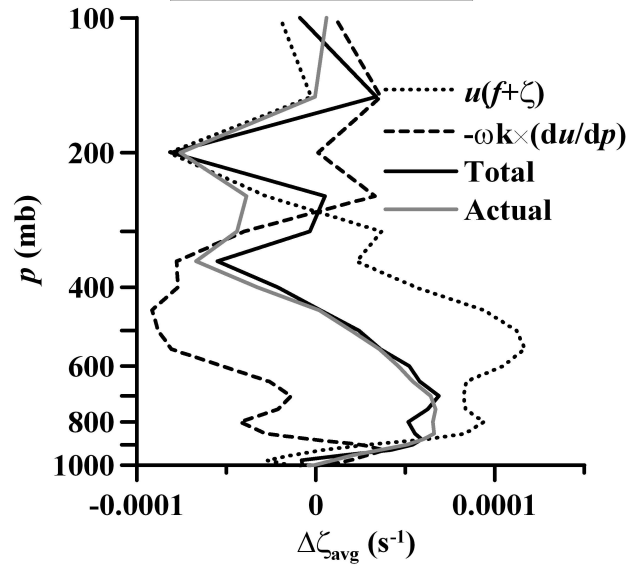

(c) $t=160 \mathrm{~h}$ to $t=164 \mathrm{~h}$

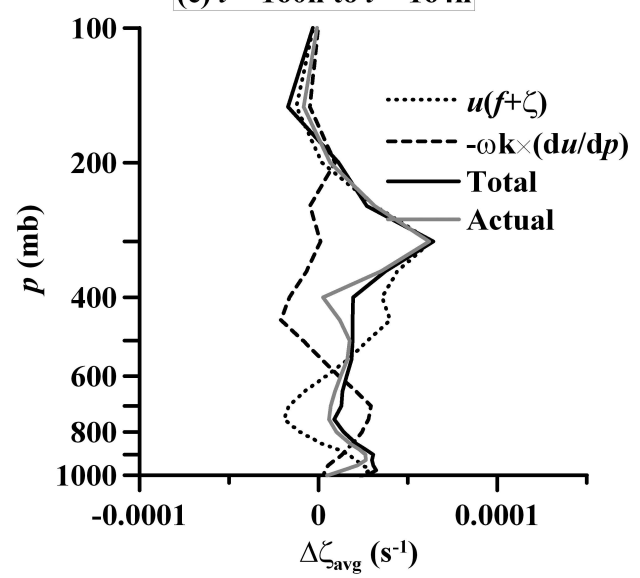

(b) $t=156 \mathrm{~h}$ to $t=160 \mathrm{~h}$

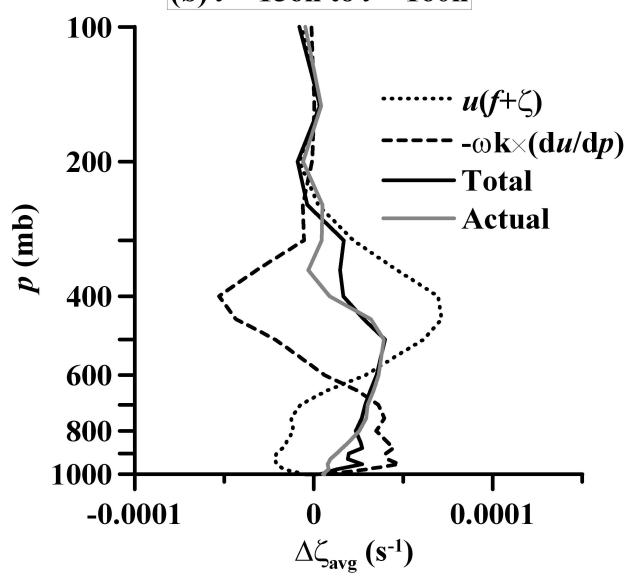

(d) $t=168 \mathrm{~h}$ to $t=172 \mathrm{~h}$

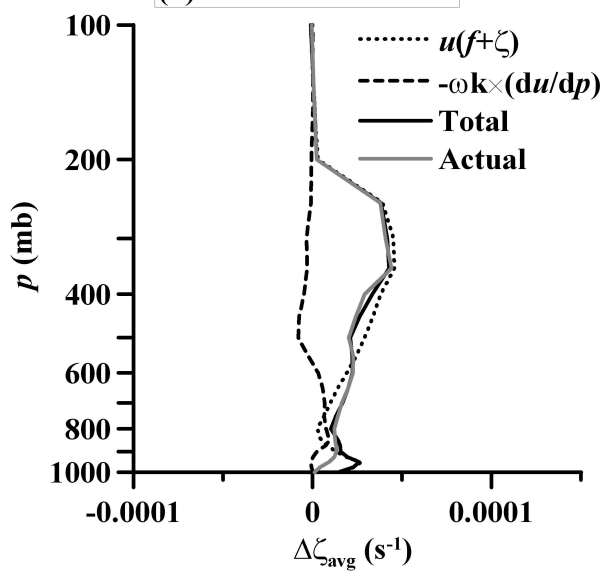

Fig. 4. Circulation budget analysis expressed as area average vorticity change for a rectangle surrounding the vortex on the $3.3-\mathrm{km}$ grid in the CAPE simulation. The time periods are as follows: (a) $152 \mathrm{~h}$ to $156 \mathrm{~h}$; (b) $156 \mathrm{~h}$ to $160 \mathrm{~h}$; (c) $160 \mathrm{~h}$ to $164 \mathrm{~h}$; and (d) $168 \mathrm{~h}$ to $172 \mathrm{~h}$, respectively. For notation, see Fig. 3.

during this time as the plots of $1000 \mathrm{hPa}$ temperature show a significant cold anomaly (as much as $10^{\circ} \mathrm{C}$ ) spreading radially outward. In mid levels, however, the flux convergence is a contributor to the intensification of cyclonic absolute vorticity. At the very end of the time period of analysis (Fig. 4d), the horizontal flux convergence again dominates the absolute vorticity budget (again, similar to Davis and Galarneau, 2009). By this time, however, the area of integration is so large that any mesoscale tilting contributes less to the areaintegrated vorticity.

\subsection{Stratiform and convective contributions to vortex intensification}

A time series of the convective and stratiform areas, within the analysis box, during the analysis intervals in the control simulation (not shown) reveals that the convection, as defined in Sect. 3.3, never occupies more than ten percent of the area of analysis. Convection initially comprises 100 percent of the total precipitation area (defined here as the sum of convective and stratiform areas), but as the stratiform precipitation area expands, the convective area decreases to as little as 7 percent of the precipitation area during the second analysis period. For the remaining analysis periods, the convection recovers to between 20 and 30 percent of the total precipitation area. By the end of the final analysis period (nearly two days after the beginning of the first), the total precipitation area has reached $60000 \mathrm{~km}^{2}$.

In the CAPE simulation, the precipitation area is considerably larger, reaching a maximum of $177000 \mathrm{~km}^{2}$. The convection starts out occupying 89 percent of the total precipitation area, but by $t=155.5 \mathrm{~h}$, it drops to less than 20 percent and then remains between 10 and 20 percent for the remaining analysis times. The convective area expands throughout the simulation and reaches over $20000 \mathrm{~km}^{2}$ by about $19 \mathrm{~h}$ after the start of the first analysis period. The stratiform area undergoes a similar expansion throughout the simulation. In the CAPE simulation, the convection provides a 
(a) $t=124.8-129.6 \mathrm{~h}$

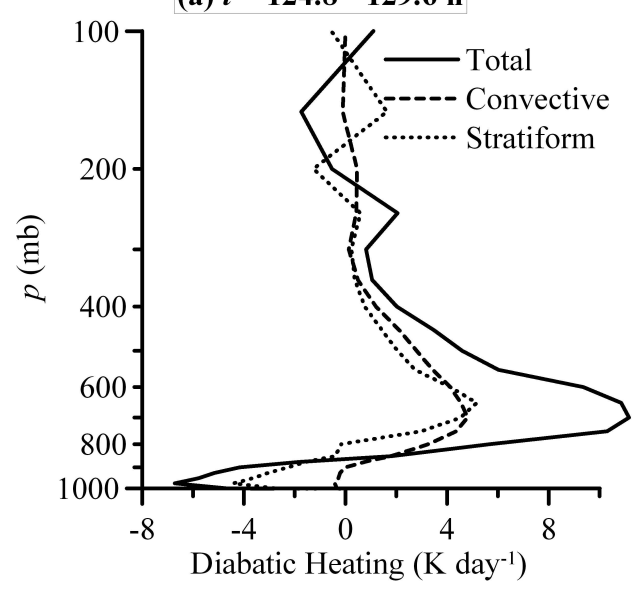

(b) $t=134.4-139.2 \mathrm{~h}$

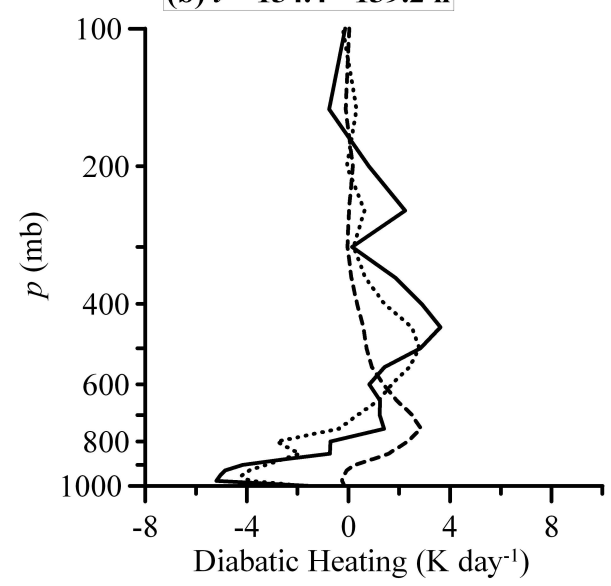

(c) $t=170.4-175.2 \mathrm{~h}$

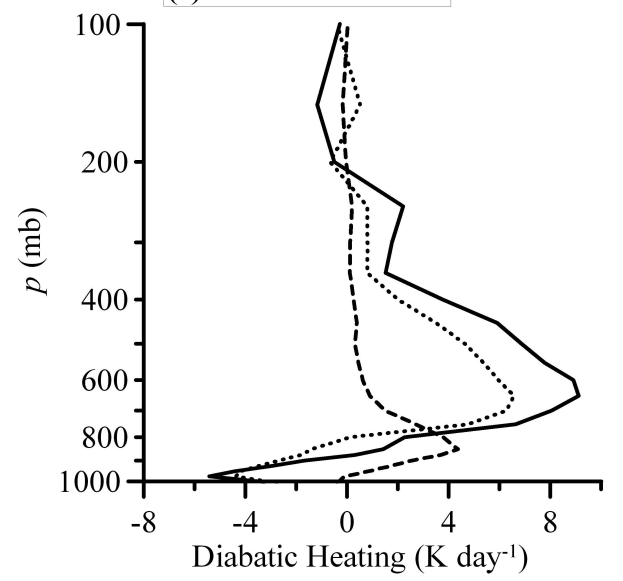

(d) $t=152-156 \mathrm{~h}$

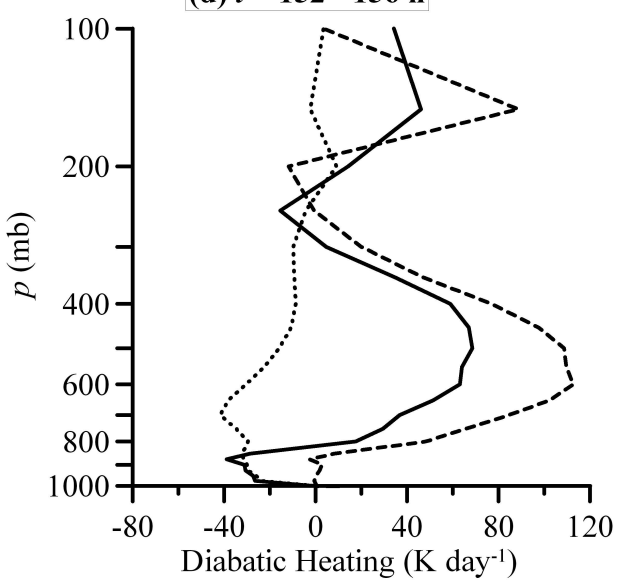

(e) $t=160-164 \mathrm{~h}$

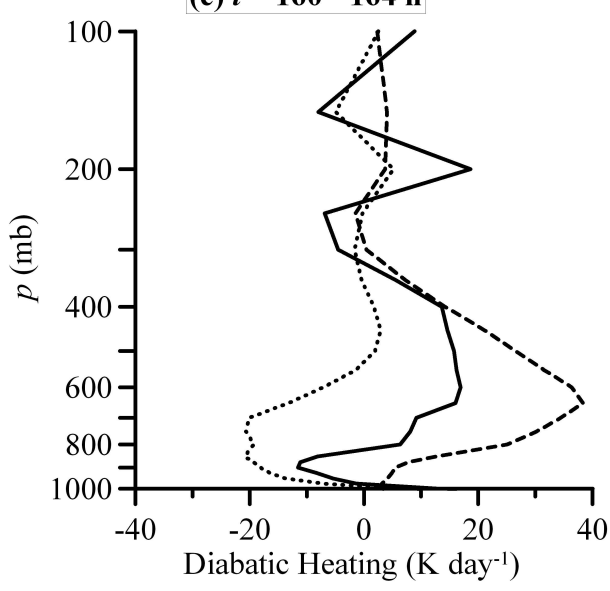

(f) $t=168-172 \mathrm{~h}$

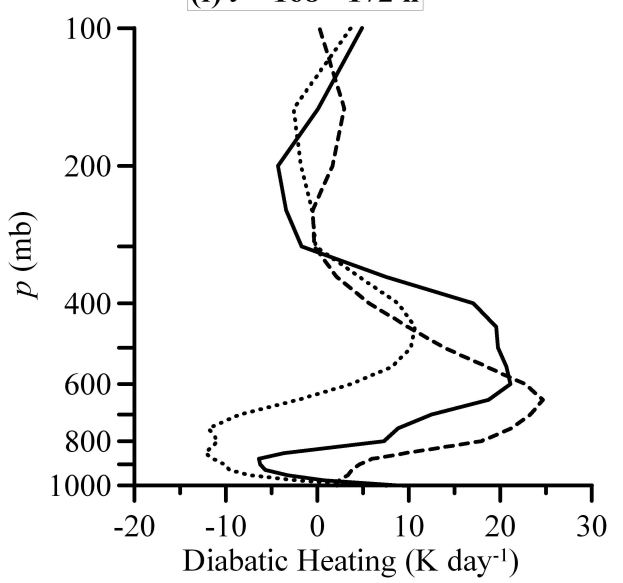

Fig. 5. Area-averaged diabatic heating, showing contributions from convective and stratiform precipitation regions in the control simulation, for a box surrounding the vortex on the $3.3-\mathrm{km}$ grid, at simulation time: (a) $124.8 \mathrm{~h}$ to $129.6 \mathrm{~h}$; (b) $134.4 \mathrm{~h}$ to $139.2 \mathrm{~h}$; and (c) $170 \mathrm{~h}$ to $175.2 \mathrm{~h}$, and for the CAPE simulation at (d) $152 \mathrm{~h}$ to $156 \mathrm{~h}$; (e) $160 \mathrm{~h}$ to $164 \mathrm{~h}$; and (f) $168 \mathrm{~h}$ to $172 \mathrm{~h}$. The area average heating rate pertains to the analysis box, which is centered on the area of the circulation and has its southern edge roughly along the line of maximum rear inflow into the system. 
much greater contribution to the MCV diabatic heating profile.

Animations of relative vorticity (not shown) suggest the dominance of tilting at smaller scales (e.g. Knievel and Johnson 2003), but on an MCV scale, the horizontal flux convergence is, by far, the dominant contributor to the vorticity change.

The evolution of the diabatic heating profiles in the control simulation is generally reflective of the evolution of the MCS (see Fig. 5). In the initial time period (Fig. 5a), the peak heating is nearly equally shared by convective and stratiform precipitation regions. Since the cooling in the stratiform area is confined to the lowest $150 \mathrm{hPa}$, the heating gradient is stronger in the stratiform region, implying the stratiform precipitation is playing the larger role in PV concentration in the lower troposphere. By $t=134.4 \mathrm{~h}$ (Fig. 5b), the stratiform precipitation region (the non-convective area experiencing a rainfall rate greater than $0.1 \mathrm{~mm} \mathrm{~h}^{-1}$ ) has expanded to occupy 80 percent of the precipitation area. Again, the diabatic cooling in the lower troposphere provides for a deeper layer of stratiform PV concentration. At this point in the simulation, the distinction between the stratiform and convective profiles is consistent with the characteristic profiles shown in Fig. 3 of Houze (1997).

During the final time period (Fig. 5c), as the system reaches a regime of steady intensification, the diabatic heating increases, and most of this diabatic heating (and therefore the PV concentration) is accomplished by the stratiform precipitation areas within the system. By this point, the diabatic heating probably reflects the moist baroclinic intensification at least as much as it is a sign of MCV dynamics. Indeed, Conzemius et al. (2007) show that, during this stage of intensification, the conversion of mean state available potential energy (APE) to eddy APE increases in tandem with the diabatic production of eddy APE. Nevertheless, the MCS dynamics are a contributor to this process by providing diabatic support to the baroclinic cyclone intensification, and balanced MCV dynamics play a part in the maintenance of convection (Conzemius et al., 2007; Trier and Davis, 2002). It is noteworthy that, during this final period, the vertical gradients in diabatic heating are largest in the lower troposphere (much like the convective profile is, see Houze, 2004), yet the precipitation is mostly stratiform. This may be symptomatic of the fact that the system has moved north into areas where the mean state has a shallower troposphere. Additionally, the system has grown upscale and therefore no longer resembles an MCS.

In the CAPE simulation (Fig. 5d through f), the diabatic heating profile is markedly different, and convective heating plays the leading role in PV concentration. At first (Fig. 5d), convection contributes essentially all of the diabatic heating, and the diabatic heating gradient is strongest between $900 \mathrm{hPa}$ and $600 \mathrm{hPa}$. The stratiform contribution to PV concentration is essentially limited to a weak layer between $700 \mathrm{hPa}$ and $450 \mathrm{hPa}$. As the stratiform area rapidly expands
(Fig. 5e), its contribution to the total becomes more significant, yet with a diabatic heating difference of $25 \mathrm{~K}$ day $^{-1}$ over the $700 \mathrm{hPa}$ to $500 \mathrm{hPa}$ layer, it still produces less PV than the convective region (nearly $40 \mathrm{~K}$ day $^{-1}$ difference in heating rate between $600 \mathrm{hPa}$ and the surface). It is thus evident that the deep convection accomplishes the bulk of the flux convergence of PV substance (see Eq. 3) until the very last period of analysis (Fig. 5f), when the stratiform diabatic heating profile becomes more consistent with the idealized profile shown in Houze (1997) and is more favorable for contributing to PV in the mid-troposphere. In this final analysis period, the convective and stratiform heating gradients are nearly equal.

Overall, convection is the dominant PV concentration mechanism in the CAPE simulation. But clearly, one $3.3 \mathrm{~km}$ simulation cannot prove that the PV concentration occurs mostly in convective updrafts within an MCS. In fact, the $3.3 \mathrm{~km}$ grid interval is relatively coarse for resolving processes essential to individual convective updrafts (e.g. Bryan et al., 2003). However, we contend that the simulation performs reasonably well at resolving processes on the scale of the MCS, and the results are believed usefully suggestive of the fact that much of the PV concentration is accomplished by deep convection. While the deep convective dominance of PV concentration is very evident in the present analysis, the verification of these results also requires a comprehensive sampling of the observed lifecycle of MCVs. The Bow Echo and MCV Experiment (BAMEX; Davis et al., 2004) has provided a wealth of observational datasets that can be used to explore this problem further.

\subsection{Trajectory analysis of the PV evolution}

In order to further illustrate the evolution of PV in the MCV, as well as the relevance of convective processes to the concentration of PV substance, we show PV horizontal cross sections at $700 \mathrm{hPa}$ (Fig. 6), which are taken from animations that were constructed from the MM5 output on the innermost (highest resolution) domain. The animation shows the creation of strong, small scale, positive and negative PV anomalies in the leading convective line of the MCS. These anomalies are associated with individual thunderstorm updrafts, whose vorticity has opposite signs due to tilting (Cram et al., 2002). After being produced in the convective region, the PV anomalies then move rearward into the stratiform precipitation region as the convective line expands outward from the MCV center (near $52^{\circ} \mathrm{N}, 16^{\circ} \mathrm{W}$ in Fig. 6a). A combination of model diffusion, vertical gradients of diabatic heating, and vorticity center mergers results in a smoother region of positive PV emerging in the central region of the MCV. For the purpose of the present discussion, we identify the central region of the MCV as the area within the $700 \mathrm{hPa}$ circulation that is free of strong negative PV anomalies and has a smooth spatial variation of $\mathrm{PV}$ relative to the outer convective region. 


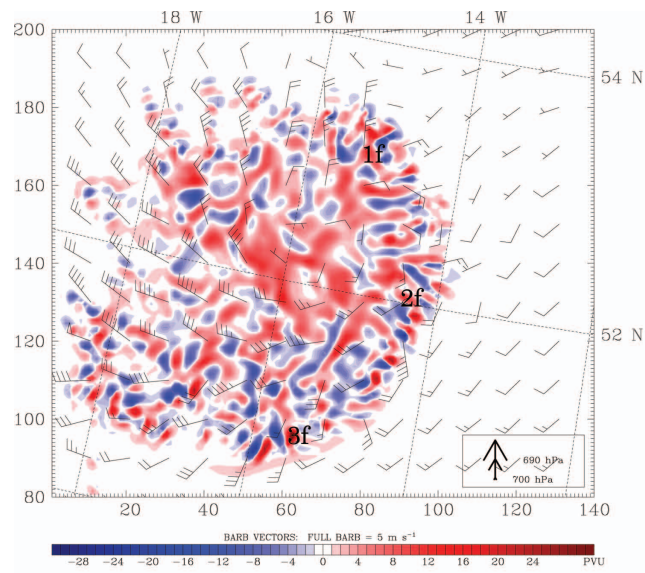

(a)

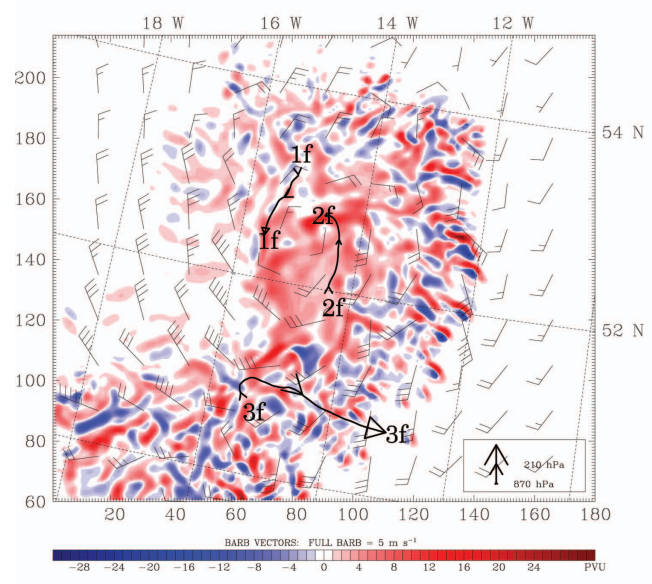

(c)

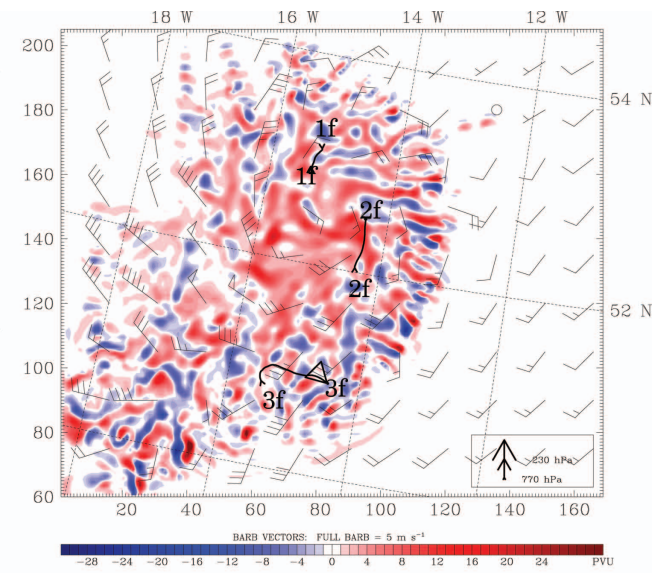

(b)

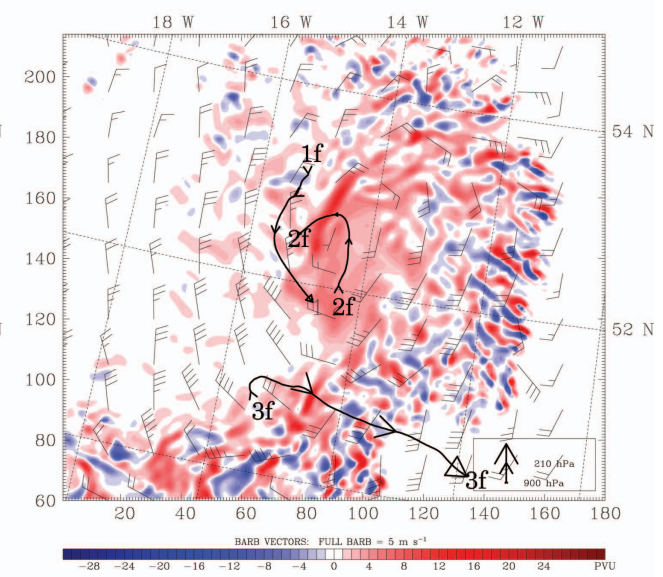

(d)

Fig. 6. Trajectory analysis and evolution of the PV $\left(\mathrm{m}^{2} \mathrm{~s}^{-1} \mathrm{~K} \mathrm{~kg}^{-1}\right)$ and winds (kt) on the $3 \mathrm{~km}$ domain of the MM5 CAPE simulation at the following times during the simulation: (a) $156.8 \mathrm{~h}$; (b) $158.4 \mathrm{~h}$; (c) $160.0 \mathrm{~h}$; and (d) $161.6 \mathrm{~h}$. The markings "1f", "2f", and "3f" refer to three trajectories originating within individual PV centers diagnosed at $t=156.8 \mathrm{~h}$ into the simulation, and the curved arrows indicate the trajectories from $t=156.8 \mathrm{~h}$ to the valid time of the PV and wind analysis. Arrows along the trajectories are drawn at intervals of $1.6 \mathrm{~h}$.

To provide more quantitative information on the above processes, we performed a trajectory analysis on three air parcels originating from separate positive PV centers identified in the animations. The analysis was performed between simulation times $t=156.8 \mathrm{~h}$ and $t=161.6 \mathrm{~h}$. The model output interval for the calculation was $8 \mathrm{~min}$ on the innermost domain. To improve the accuracy of trajectory calculations, we used a trajectory time step of $80 \mathrm{~s}$. The Read, Interpolate, and Plot (RIP) program (part of the MM5 analysis system) was used to calculate the trajectories.

At $t=156.8 \mathrm{~h}$ (Fig. 6a), the originating points of these three trajectories, labeled " $1 \mathrm{f}$ ", " $2 \mathrm{f}$ ", and " $3 \mathrm{f}$ ", are shown (we use this same labeling to refer both to the trajectories and to the air parcels following them). By $t=158.4 \mathrm{~h}$ (Fig. 6b), as the convection expands outward, the three identified parcels all initially move rearward relative to the leading convective line and begin to rotate cyclonically about a common center.
Parcel $3 \mathrm{f}$ ascends and moves east-southeastward, however, eventually exiting the MCS in the strong, westerly upper tropospheric flow (Fig. 6d). At $t=160.0 \mathrm{~h}$ (Fig. 6c), parcels $1 \mathrm{f}$ and $2 \mathrm{f}$ continue to rotate about the center of the MCV and are now essentially part of the central circulation of the MCV. By $t=161.6 \mathrm{~h}$ (Fig. 6d), parcel $2 \mathrm{f}$ has moved very close to the center of the MCV and has begun to move toward the southwest. Parcel $1 \mathrm{f}$ has rotated cyclonically around the western side of the MCV center. The analyses presented in Figs. 4 and 5 show that the rotation comes about primarily due to the flux convergence of absolute vorticity as the system rapidly intensifies due to the diabatic heating.

Overall, the illustration shows that PV anomalies along trajectories 1f and $2 \mathrm{f}$ are created by the leading edge convection, then move rearward and become somewhat less intense. It must be noted that parcel $2 \mathrm{f}$ descended from its initial altitude near $3000 \mathrm{~m}$ a.g.l. (Fig. 6a) to a final altitude 


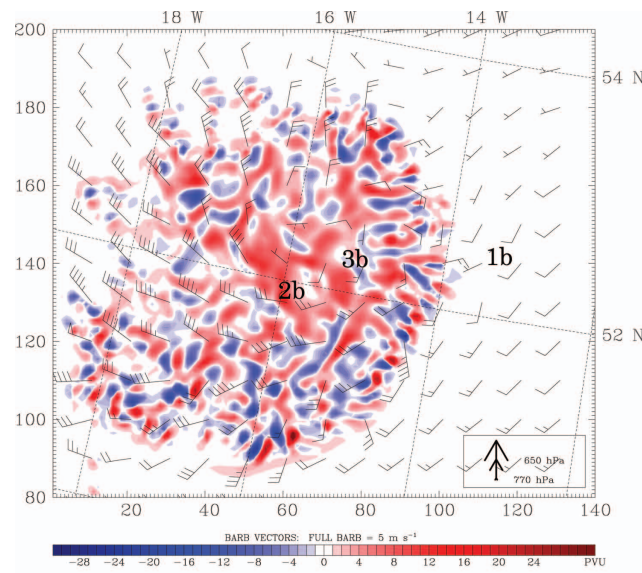

(a)

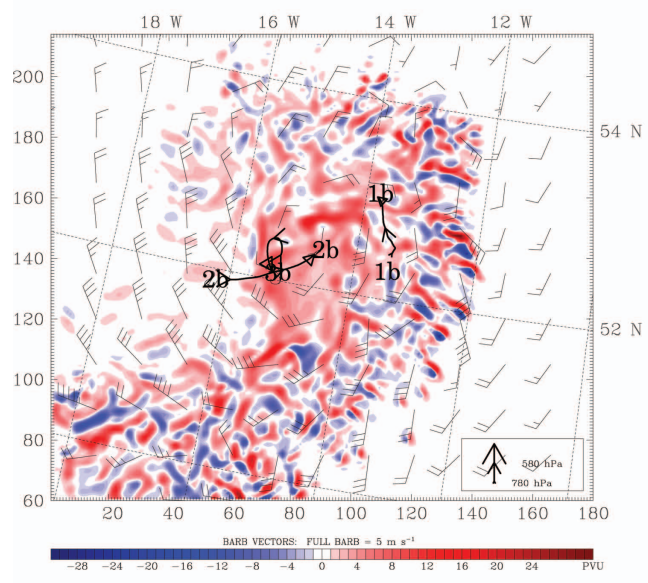

(c)

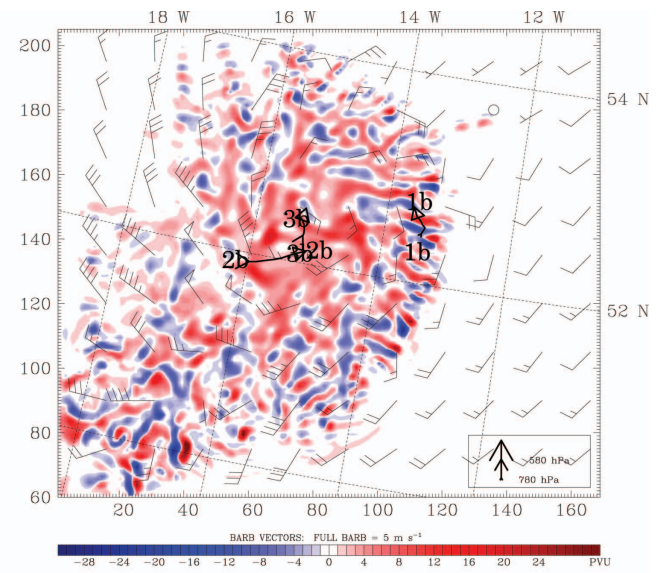

(b)

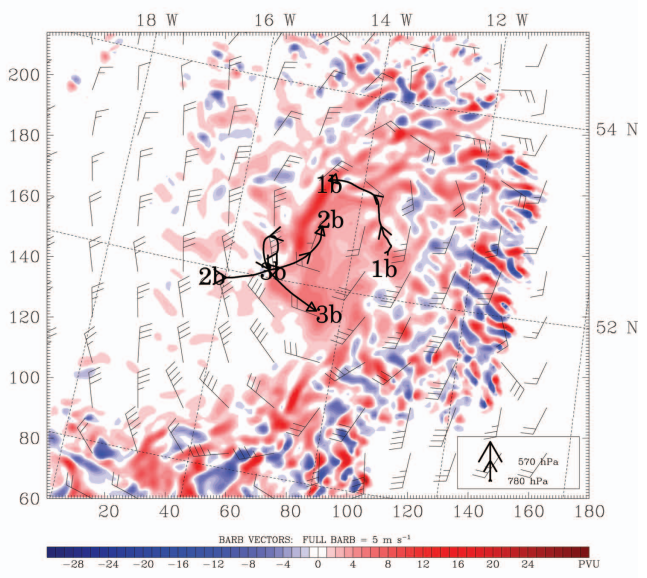

(d)

Fig. 7. Back-trajectory analysis and evolution of the PV $\left(\mathrm{m}^{2} \mathrm{~s}^{-1} \mathrm{~K} \mathrm{~kg}^{-1}\right)$ and winds (kt) on the $3 \mathrm{~km}$ domain of the MM5 CAPE simulation at the following times during the simulation: (a) $156.8 \mathrm{~h}$; (b) $158.4 \mathrm{~h}$; (c) $160.0 \mathrm{~h}$; and (d) $161.6 \mathrm{~h}$. The markings " $1 \mathrm{~b}$ ", "2b", and " $3 \mathrm{~b}$ " refer to three trajectories ending in individual PV centers diagnosed at $t=161.6 \mathrm{~h}$ into the simulation. The back-trajectories were calculated from $t=161.6 \mathrm{~h}$ to $t=156.8 \mathrm{~h}$, and the curved arrows indicate the same back-trajectories, forward, from $t=156.8 \mathrm{~h}$ to the valid time of the PV and wind analysis. Arrows along the trajectories are drawn at intervals of $1.6 \mathrm{~h}$.

of approximately $1000 \mathrm{~m}$ a.g.l., so its final location is below the $700 \mathrm{hPa}$ level shown in Fig. 6d. Parcel 1f remained near $3000 \mathrm{~m}$ a.g.l., while parcel $3 \mathrm{f}$ reached an altitude over $11000 \mathrm{~m}$ a.g.l. by the end of the analysis period.

Since two of the three trajectories ended at points horizontally or vertically displaced from the MCV central region shown in Fig. 6, we also calculated backward trajectories for three points within the $700 \mathrm{hPa} \mathrm{MCV}$ central region at $t=161 \mathrm{~h}$ (Fig. 7). The three end points chosen were from a positive PV anomaly in the northern part of the MCV (1b), a location at the center of the circulation (2b), and a positive $\mathrm{PV}$ anomaly from the southern portion of the MCV (3b). See Fig. $7 \mathrm{~d}$ for the identified locations of these three centers.

The backward trajectory calculations show that parcel $2 b$ and parcel $3 \mathrm{~b}$ were already near the center of the MCS at the initial time of $t=156.8 \mathrm{~h}$ (Fig. 7a). Both these parcels rotated cyclonically around each other over the entire period of analysis. Parcel $2 b$ started at an altitude of $3600 \mathrm{~m}$ a.g.l., and parcel 3b started at an altitude of $3200 \mathrm{~m}$ a.g.l.. Meanwhile, parcel $1 \mathrm{~b}$ started from an altitude of $2300 \mathrm{~m}$ a.g.l. at a location east of the MCS, entered the MCS in the convective region (Fig. 7b), ascended to an altitude of $4500 \mathrm{~m}$ a.g.l. at $t=157.9 \mathrm{~h}$, then gradually descended to its final altitude as it became entrained into the central region of the MCV (Fig. 7d). All three parcels have rotated cyclonically around a common center of circulation.

Further insight into the role of stratiform and convective processes can be gained by tracking the evolution of PV along these trajectories. For this final analysis, we concentrated only on those trajectories that began $(t=156.8 \mathrm{~h})$ outside the MCV circulation and ended $(t=161.6 \mathrm{~h})$ in the central MCV circulation at an altitude between $2500 \mathrm{~m}$ and 


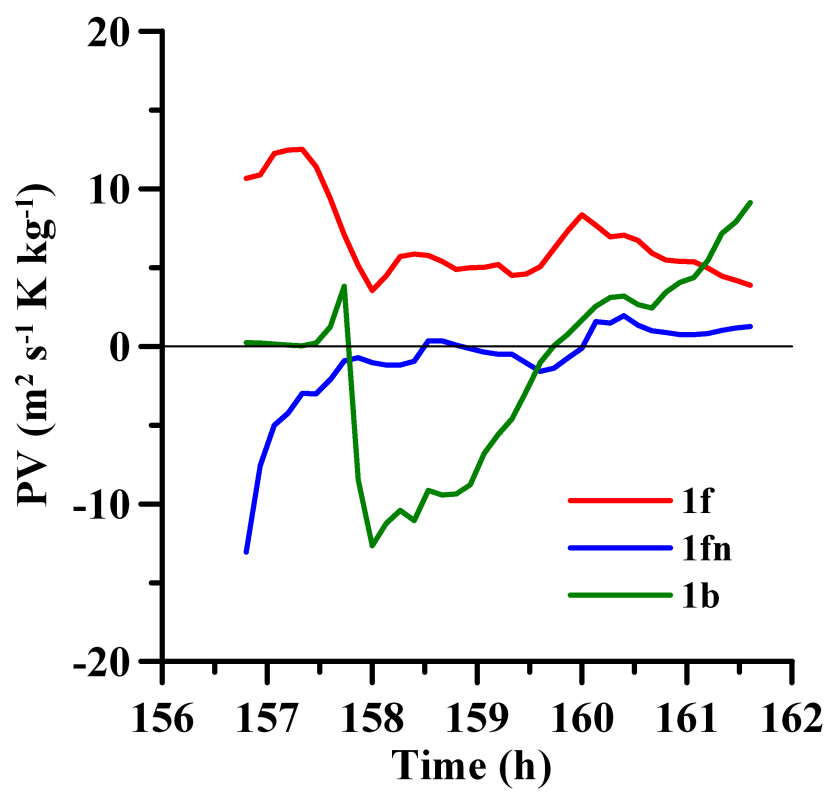

Fig. 8. Evolution of $\mathrm{PV}\left(\mathrm{m}^{2} \mathrm{~s}^{-1} \mathrm{~K} \mathrm{~kg}^{-1}\right)$ along parcel trajectory 1f (positive anomaly identified in Fig. 6), the trajectory originating from the nearest negative PV center (1fn), and the back-trajectory $1 \mathrm{~b}$ (identified in Fig. 7) according to the simulation time. For locations of these trajectories at various times, refer to Figs. 6 and 7 (trajectory $1 \mathrm{fn}$ is not shown but lies very close to trajectory $1 \mathrm{f}$ ).

3500 m a.g.1.. Because our forward trajectories originated only from large magnitude positive PV anomalies, the analysis would be biased to conclude that convection is the primary contributor to positive PV anomalies in the MCV central region. Thus, we also calculated, for each of the three trajectories in Fig. 6, the corresponding trajectories originating from the nearest negative PV anomaly (1fn, $2 \mathrm{fn}$, and $3 \mathrm{fn}$, respectively - not shown). Trajectories $1 \mathrm{fn}$ and $2 \mathrm{fn}$ remained very close to trajectories $1 \mathrm{f}$ and $2 \mathrm{f}$, but only $1 \mathrm{fn}$ finished between $2500 \mathrm{~m}$ and $3500 \mathrm{~m}$ a.g.1.. Thus, for our PV tracking analysis, we focused on trajectories $1 \mathrm{~b}, 1 \mathrm{f}$, and $1 \mathrm{fn}$.

The results (Fig. 8) show that large PV fluctuations occur whenever the trajectories are located within the MCS. For each of the trajectories, the most rapid PV change occurs in the earliest time intervals, when the parcels are located within the leading line of convection. Of particular interest is trajectory $1 \mathrm{fn}$. PV values increase very rapidly through $t=157.5 \mathrm{~h}$. The values then fluctuate but experience a net increase between $t=157.5 \mathrm{~h}$ and $t=161.6 \mathrm{~h}$. Trajectory $1 \mathrm{~b}$ initially experiences some strong positive PV concentration as it enters the convective region, but its $\mathrm{PV}$ decreases very rapidly over a 15 min time interval ending at $t=158 \mathrm{~h}$. Thereafter, its PV experiences a rather steady and strong increase.

The overall picture that emerges from Figs. 5, 6, 7, and 8 is that the diabatic heating gradients, and hence PV concentration, in the CAPE simulation are clearly strongest in the convective region of the MCS. Because the tilting processes in convective updrafts generate dipoles of vertical vorticity, the strong diabatic heating gradients in the convective region are used for the concentration of both positive and negative PV anomalies. Thus, it may be difficult to conclude, based on Figs. 6, 7, and 8, that the ultimate source of positive PV anomalies in the MCV central region is primarily from the leading convective line. Regardless, the intense positive and negative dipoles, which require tilting processes, can be expected to largely cancel. Although an exhaustive attribution of $\mathrm{PV}$ concentration by various processes is obviously a complex procedure, the largest source of the positive PV anomaly in the MCV central region has to come from the flux convergence of absolute vorticity (the majority of which is planetary vorticity in the initial condition) acting in concert with the convective diabatic heating.

\section{Conclusions}

In this paper, we have clarified several outstanding issues concerning the predominant mechanism of vorticity generation in mesoscale convective vortices (MCVs) in weak to modest baroclinic environments with nonzero Coriolis parameter. Using idealized mesoscale numerical simulations of MCV development, we examined the vertical vorticity budgets in order to quantify the contributions of flux convergence of absolute vorticity versus tilting in the generation of MCV vorticity. We have examined also the corresponding diabatic heating profiles. By partitioning the diabatic heating between convective and stratiform regions, we elucidated the respective roles of convective and stratiform precipitation in the concentration of potential vorticity (PV) substance.

The analyses indicated that the horizontal flux convergence of vertical vorticity is the dominant mechanism for the spin-up and intensification of mid-level absolute vorticity. Correspondingly, diabatic heating and circulation budgets demonstrated that the vertical gradient of diabatic heating is supportive of low- to mid-level concentration of PV substance. When the CAPE is representative of warm season conditions, and during the early stages of MCV development, convective precipitation plays the dominant role in the concentration of PV. During later stages in the MCV life cycle, the contribution from stratiform processes increases. In contrast, in an environment with near zero CAPE, stratiform precipitation is the dominant contributor to $\mathrm{PV}$ concentration.

Trajectory analyses and plots of PV on the innermost grid of the simulations revealed that the convective region has strong PV anomalies of positive and negative sign. If we use the absolute vorticity diagnostics as a proxy for the evolution of PV substance, then we find a cyclonic vorticity rich environment favors the emergence of a cyclonic PV anomaly behind the leading convective line.

Acknowledgements. This work was conducted under the support of NSF grant ATM-0305412 and the US Naval Postgraduate School in Monterey, California, USA. We would also like to thank David 
Raymond, Chris Davis, and an anonymous reviewer for their constructive comments towards improving the analysis techniques in this manuscript.

Edited by: H. Wernli

\section{References}

Bartels, D. L. and Maddox, R. A.: Midlevel cyclonic vortices generated by mesoscale convective systems. Mon. Weather Rev., 119, 104-118, 1991.

Brandes, E. A.: Evolution and structure of the 6-7 May 1985 mesoscale convective system and associated vortex. Mon. Weather Rev., 118, 109-127, 1990.

Braun, S. A., Montgomery, M. T. , Mallen, K., and Reasor, P.: Simulation and interpretation of the genesis of tropical storm Gert (2005) as part of the NASA Tropical Cloud Systems and Processes Experiment, J. Atmos. Sci., 66, DOI 10.1175/2009JAS3140.1, 2009.

Bryan, G. H., Wyngaard, J. C., and Fritsch, J. M.: Resolution requirements for the simulation of deep moist convection. Mon. Weather Rev., 131, 2394-2416, 2003.

Conzemius, R. J., Moore, R. W., Montgomery, M. T., and Davis, C. A.: Mesoscale convective vortex formation in a weakly sheared moist neutral environment. J. Atmos. Sci., 64, 1443-1466, 2007.

Cram, T. A., Montgomery, M. T., and Hertenstein, R. F. A.: Early evolution of vertical vorticity in a numerically simulated idealized convective line, J. Atmos. Sci., 59, 2113-2127, 2002.

Davis, C. A., and Galarneau, T. J., Jr.: The vertical structure of mesoscale convective vortices, J. Atmos., Sci., 66, 686-704, 2009.

Davis, C. A., and Trier, S. B.: Cloud-resolving simulations of mesoscale vortex intensification and its effect on a serial mesoscale convective system, Mon. Weather Rev., 130, 28392858, 2002.

Davis, C. A., Atkins, N., Bartels, D., Bosart, L., Coniglio, M., Bryan, G., Cotton, W., Dowell, D., Jewett, B., Johns, R., Jorgensen, D., Knievel, J., Knupp, K., Lee, W.-C., Mcfarquhar, G., Moore, J., Przybylinski, R., Rauber, R., Smull, B., Trapp, R., Trier, S., Wakimoto, R., Weisman, M., and Ziegler, C.: The bow echo and MCV experiment: observations and opportunities, B. Am. Meteor. Soc., 85, 1075-1093, 2004.

Fovell, R. G. and Ogura, Y.: Numerical simulation of a midlatitude squall line in two dimensions. J. Atmos. Sci., 45, 3846-3879, 1988.

Fritsch, J. M., Murphy, J. D., and Kain, J. S.: Warm core vortex amplification over land. J. Atmos. Sci., 51, 1780-1807, 1994.
Grell, G. A., Dudhia, J., and Stauffer, D. R.: A description of the fifth-generation Penn State/NCAR mesoscale model (MM5). NCAR Tech. Note NCAR/TN-398_STR, 117 pp., 1994.

Haynes, P. H. and McIntyre, M. E.: On the evolution of vorticity and potential vorticity in the presence of diabatic heating and frictional or other forces, J. Atmos. Sci., 44, 828-841, 1987.

Hertenstein, R. F. A. and Schubert, W. H.: Potential vorticity anomalies associated with squall lines, Mon. Weather Rev., 119, 1663-1672, 1991.

Houze Jr., R. A.,: Stratiform precipitation in regions of convection: A meteorological paradox?, B. Am. Meteor. Soc., 78, 21792196, 1997.

Houze Jr., R. A.,: Mesoscale convective systems. Rev. Geophys., 42, RG4003, doi:10.1029/2004RG000150, 2004.

Johnson, R. H., and Bartels, D. L.: Circulations associated with a mature-to-decaying midlatitude mesoscale convective system. Part II: upper-level features. Mon. Weather Rev., 120, 13011321, 1992.

Kirk, J. R.: A phase-plot method for diagnosing vorticity concentration mechanisms in mesoscale convective vortices. Mon. Weather Rev., 135, 801-820, 2007.

Knievel, J. C. and Johnson, R. H.: A scale-discriminating vorticity budget for a mesoscale vortex in a midlatitude, continental mesoscale convective system. J. Atmos. Sci., 60, 781-794, 2003.

Raymond, D. J. and Jiang, H.: A theory for long-lived mesoscale convective systems. J. Atmos. Sci., 47, 3067-3077, 1990.

Rotunno, R.: On the evolution of thunderstorm rotation, Mon. Weather Rev., 109, 577-586, 1981.

Skamarock, W. C., Weisman, M. L., and Klemp, J. B.: Threedimensional evolution of simulated long-lived squall lines, J. Atmos. Sci., 51, 2563-2584, 1994.

Tao, W.-K., Simpson, J., Sui, C. H., B. Ferrier, B., Lang, S., Scala, J., Chou, M. D., and Pickering, K.: Heating, moisture, and water budgets of tropical and midlatitude squall lines: comparisons and sensitivity to longwave radiation. J. Atmos. Sci., 50, 673-690, 1993.

Tory, K. J., Montgomery, M. T., Davidson, N. E., and Kepert, J. D.: Prediction and diagnosis of tropical cyclone formation in an NWP system. Part II: a diagnosis of tropical cyclone Chris formation, J. Atmos. Sci., 63, 3091-3113, 2007.

Trier, S. B. and Davis, C. A.: Influence of balanced motions on heavy precipitation within a long-lived convectively generated vortex, Mon. Weather Rev., 130, 877-899, 2002.

Weisman, M. L. and Davis, C. A.: Mechanisms for the generation of mesoscale vortices within quasi-linear convective systems, J. Atmos. Sci., 55, 2603-2622, 1998. 